Décadrages Décadrages

cınéma, à travers champs Cinéma, à travers champs

37-38 | 2018

Lionel Rogosin

\title{
Interventions au Festival International du Nouveau Cinéma de Pesaro, 1965
}

\section{Lionel Rogosin}

Traducteur : Faye Corthésy

\section{OpenEdition \\ Journals}

\section{Édition électronique}

URL : https://journals.openedition.org/decadrages/1266

DOI : $10.4000 /$ decadrages. 1266

ISSN : 2297-5977

\section{Éditeur}

Association Décadrages

\section{Édition imprimée}

Date de publication : 1 mars 2018

Pagination : 153-161

ISBN : 978-2-9700963-4-4

ISSN : 2235-7823

\section{Référence électronique}

Lionel Rogosin, «Interventions au Festival International du Nouveau Cinéma de Pesaro, 1965 », 
Lionel Rogosin

(traduction de l'américain et introduction par Faye Corthésy)

\section{Interventions au Festival International du Nouveau Cinéma de Pesaro, 1965}

Ces deux textes ont été commandés par les organisateurs de la première édition du Festival International du Nouveau Cinéma (Mostra Internazionale del Nuovo Cinema) de Pesaro, qui a lieu du 29 mai au 6 juin 1965. En marge des projections de films, le festival italien organise deux "séminaires » à cette occasion: le premier sur la distribution internationale des œuvres et le second sur les liens entre la critique et le «nouveau cinéma», qui prend la forme d'une table ronde. Le texte du premier règlement de la jeune manifestation, organisée sous l'égide de I'Institut National du Spectacle italien, annonce en effet que celle-ci a non seulement pour objectif de valoriser des films de réalisateurs «débutants» et de "présenter un vaste panorama des innovations les plus significatives du nouveau cinéma», mais aussi de "promouvoir, parmi les représentants du nouveau cinéma, des rencontres, un échange réciproque de leurs expériences et des débats», afin de "favoriser la diffusion et la mise en circulation des œuvres présentées » ${ }^{1}$.

Lino Micchichè, qui dirige le festival, invite dans un premier temps Rogosin à présenter son film récemment achevé, Good Times, Wonderful Times (1965), dans la "section culturelle et d'informations» (le réalisateur étatsunien étant trop expérimenté pour figurer dans la section principale consacrée aux premières œuvres, ou aux «première[s] tentative[s] de cinéma $d$ 'auteur de la part de metteurs en scène ${ }^{2}{ }^{2}$ ). Après une série d'échanges, la présentation du film est annulée, suite à sa sélection au Festival international du film de Venise, qui se déroule entre fin août et début septembre et qui exige l'exclusivité de ses premières. Micchichè insiste toutefois sur l'importance de la participation de Rogosin aux deux séminaires car, comme il l'écrit au réalisateur, il "pourrait très bien représenter le cinéma en dehors d'Hollywood [off-Hollywood] »: "les raisons pour lesquelles nous organisons ce festival [...] sont similaires à votre combat personnel au sein du Cinéma et avec celui-ci », conclut-il ${ }^{3}$. L'intérêt des organisateurs pour Rogosin
1 Istituto Nazionale dello Spettacolo, «I Mostra Internazionale del Nuovo Cinema» (dépliant), Rome, 1965, archives privées de Lionel Rogosin, Angers, France (ALR).

2 Ibid.

3 Lettre de Lino Micchichè à Lionel Rogosin, Rome, 26 février 1965, ALR [ma trad.]. 
4 A ce sujet, voir ma contribution à ce numéro, «Du côté de la distribution ».

5 Festival International du Nouveau $\mathrm{Ci}$ néma de Pesaro, "Round Table: Criticism and New Cinema", [Rome], circa début 1965, ALR [ma trad.]. Une liste de quarantehuit noms de cinéastes, producteurs, distributeurs et gérants de salle internationaux (en majorités européens, mais aussi asiatiques, d'Amérique du Sud et du Nord), divisée en deux commissions, accompagne ces feuillets.

6 Lettre de Lionel Rogosin à Mino Argentieri, [New York], 21 mai 1965, ALR [ma trad.]. Argentieri avait demandé à Rogosin une version écrite de son intervention en vue de la traduire en français et en italien avant le début du festival. Dans sa lettre, Rogosin précise qu'il a eu «beaucoup de peine à l'écrire», et qu'il est "au milieu d'une recherche sur l'ensemble du problème [et] espère avoir une contribution plus complète quand il arrivera à Pesaro». C'est pourtant la première version de sa contribution qui sera publiée et traduite en italien, avec celle des autres participants. Pour cette traduction, je me suis basée sur la version anglaise du texte de Rogosin conservée dans ses archives, et non sur la traduction italienne qui paraît, sans titre, dans la revue Marcatré ( ${ }^{\circ} 19-22$, avril 1966, Lerici Editori), et qui est rééditée dans le recueil $P e r$ una nuova critica: I convegni pesaresi 1965-1967 (Venise, Marsilio Editori, 1989, pp. 125-129), sous le titre «Nouveaux marchés pour un nouveau cinéma» ["Nuovi mercati per un nuovo cinema»].

7 «Round Table: Criticism and New Cinema », op. cit. [ma trad.]. s'explique à la fois par les méthodes de production et de réalisation de ses deux premiers films, On the Bowery (1956) et Come Back, Africa (1959), qui ont circulé en Europe et sont proches du cinéma défendu par le festival, et par son engagement plus large pour la médiatisation des films indépendants, notamment via sa participation au New American Cinema Group et sa gestion du Bleecker Street Cinema, la salle qu'il a ouverte à New York en $1960^{4}$.

Le premier texte, traduit à partir d'un feuillet dactylographié conservé dans les archives privées de Rogosin, n'a jamais été publié (mais a peut-être circulé lors du festival). II s'agit de notes en vue de son intervention lors de la rencontre concernant la distribution internationale des "films modernes». Le but de cette rencontre est présenté en ces termes par le festival: "[le séminaire] étudiera la possibilité d'étendre la distribution internationale des œuvres de jeunes réalisateurs qui, trop fréquemment, restent dans l'ombre; il reviendra aussi sur la question de la coopération entre les réalisateurs indépendants ${ }^{5}$. L'intervention préparée par Rogosin répond à ces directives de façon critique, en interrogeant l'utilité même d'une telle réunion. Son discours prend ensuite un ton plus constructif et rassembleur, en appelant à l'engagement collectif des cinéastes pour la construction d'un cinéma qui soit en dialogue avec la société, dans le contexte de la guerre froide.

Le second texte consiste en un «brouillon » de son intervention à la table ronde sur les rapports entre la critique et le nouveau cinéma que Rogosin envoie au critique Mino Argentieri, qui fait partie du comité d'organisation du festival ${ }^{6}$. La table ronde réunit des cinéastes et des critiques de cinq pays considérés alors comme des centres importants dans le développement du «nouveau cinéma» auquel le festival est consacré: outre l'Italie qui, dans ce contexte, compte logiquement le plus de participants (Pier Paolo Pasolini, Paolo et Vittorio Taviani, Mino Argentieri, Gianni Toti, Giulio C. Castello), la France (Alain Jessua, Paul-Louis Thirard, Marcel Martin), l'Angleterre (Lindsay Anderson, Geoffrey Nowell-Smith, Alan Lovell), la Tchécoslovaquie (Milos Forman, Jaromil Jires, Drahomira Olivova), et les Etats-Unis (le critique au Village Voice et à Film Culture Andrew Sarris accompagne Rogosin) sont représentés. Dès le départ, il est prévu de publier les actes de cette table ronde, dont le but est d'une part d'informer les participants et a posteriori les lecteurs des «tendances majeures dans le cinéma contemporain », et d'autre part de «parvenir à une fertilisation croisée des contributions des réalisateurs et des critiques ${ }^{7}$. 
Argentieri avait précisé à Rogosin que son intervention devrait «aider le public et les participants à la discussion à comprendre dans quelle mesure il y a en Amérique [...] un dialogue entre critiques et cinéastes engagés dans un renouvellement du contenu et du langage des films »; il l'encourageait à proposer une «intervention provocante comportant au besoin des observations polémiques ${ }^{8}$. Rogosin établit un constat intransigeant dans la première partie de son intervention, avant de s'aligner sur la position de Jonas Mekas, lui-même critique et défenseur du «nouveau cinéma américain », qui s'oppose frontalement aux écrits des critiques de cinéma dans la presse quotidienne américaine ${ }^{9}$.

L'intervention et la participation de Rogosin semblent avoir suscité l'enthousiasme puisqu'à la conclusion du festival, il est élu à la présidence d'un comité provisoire pour établir un Centre International des Films Modernes (International Modern Films Center) lié au festival et basé à Rome - un projet qui ne sera toutefois que de courte durée ${ }^{10}$.

\section{Adresse à Pesaro}

Cette ASSEmbléE EST TRÈS ImPORTANTE et pourrait changer le monde - mais elle ne le fera probablement pas, car nous nous contenterons de parler. Peut-être ceci est-il positif, et quelque chose en ressortira, car nos discussions seront si ennuyantes que l'un d'entre nous va se précipiter en dehors de cette assemblée et agir. En tant que groupe, nous souffrons du même problème que celui dont la société a toujours souffert: les hommes de bonne intention sont faibles comparés à ceux qui travaillent pour leurs propres intérêts. Nous devons devenir plus forts et sortir de nos tours d'ivoire. Les artistes, les penseurs, les professeurs doivent entrer en dissidence et prendre des mesures pour que quelque chose se passe.

Nous restons assis ici à ne rien faire et parlons vainement - en fait, nous sommes trop complaisants à l'égard de l'état tragique de la distribution de films. Comment pouvons-nous être aussi complaisants? Que pensons-nous qu'est l'art? Ce n'est ni gribouiller sur les murs ni jouer un air agréable sur une guitare - c'est l'avancée de la civilisation. L'art est un témoignage de la vérité. Nous ne pouvons pas être complaisants.
8 Lettre de Mino Argentieri à Lionel Rogosin, Rome, 30 avril 1965, ALR [ma trad.].

9 Voir le chapitre "The 〈Flaming) Freedom of Jonas Mekas", dans Raymond J. Haberski, Freedom to Offend: How New York Remade Movie Culture, Lexington, University Press of Kentucky, 2007, pp. 119-151.

10 A ce sujet, voir "Du côté de la distribution», op. cit. 
11 Rogosin fait référence ici à la programmation d'On the Bowery dans le cadre de la deuxième série de films présentée en septembre 1956 au National Film Theatre, à Londres, sous l'étiquette du «Free Cinema». Le premier programme avait réuni des films récents réalisés en Angleterre par les cinéastes organisateurs (O'Dreamland de Lindsay Anderson [1953], Momma Don't Allow de Karel Reisz et Tony Richardson [1955] et Together de Lorenza Mazzetti [1956]); le deuxième, d'après le prospectus de présentation, entend montrer que «ce n'est pas seulement dans ce pays que le Free Cinema est nécessaire ou existe [...]. Partout, c'est une véritable lutte que de réaliser des films honnêtes, et un combat acharné pour les faire circuler. » («Free Cinema Two », 1956, ALR [ma trad.]) Le film de Rogosin, présenté comme « un exemple exceptionnel de ce que [les organisateurs] entendent par Free Cinema», par «sa technique réaliste audacieuse, sa franchise et sa vision poétique et compassionnelle», est projeté avec Neighbours de Norman McLaren (Can., 1952) et Le Sang des bêtes de Georges Franju (Fr., 1949).

12 En mars 1959, la sixième série de films au National Film Theatre est aussi la dernière. Constatant à la fois les accomplissements du «mouvement» et les obstacles liés à la production et à la distribution de films «hors du système ", les organisateurs annoncent «Nous n'abandonnerons pas [nos] convictions, ni [nos] tentatives de les mettre en pratique. Mais nous sentons que ce mouvement, sous cette bannière spécifique, a accompli sa tâche. » (Lindsay Anderson, John Fletcher, Walter Lassally et Karel Reisz, «Free Cinema 6: The Last Free Cinema » [1959], dans Scott MacKenzie [éd.], Film Manifestos and Global Cinema Cultures: A Critical Anthology, Berkeley/Los Angeles/Londres, University of California Press, 2014, pp. 150-151 [ma trad.]).
Nous devrions vivre chaque jour, comme Dostoïevski l'a dit, «...comme si nous venions d'échapper au bourreau». Nous vivons l'un des moments les plus cruciaux de l'histoire et il n'y a aucune raison de nous montrer si complaisants. Ce pourrait être la début d'une nouvelle et grande époque, la fin de la pauvreté et de la misère, la fin de la famine, la fin de la guerre. A l'inverse, ce pourrait être la fin de la civilisation telle que nous la connaissons. Chaque contribution, chaque action, est importante - et le cinéma ne se situe pas en dehors de cette lutte. Il n'en est pas séparé.

J'ai découvert le Free Cinema anglais avec beaucoup d'intérêt. Mon premier film, On the Bowery, a connu sa première diffusion publique sous ses auspices ${ }^{11}$. A cette époque, j'étais très intéressé par ce que faisaient Lindsay Anderson et les autres nouveaux réalisateurs anglais. Le free cinema en Angleterre n’a pas échoué; nous avons échoué12. Nous n’avons pas réussi à soutenir ce cinéma qui n'aurait pas pu survivre sans un free cinema équivalent dans chaque pays. C'est ce dont nous avons besoin maintenant.

J'en appelle ici aux producteurs, aux réalisateurs et aux distributeurs. Faisons don de notre temps, de notre argent, ou des deux. Une heure par jour, par semaine ou par mois. Fixons-nous chacun une tâche et apportons notre propre contribution.

Il est temps de renverser cette situation qui a perduré à travers les âges. A l'opposé de l'homme d'affaires, qui ne comprend pas l'âme humaine, qui ne comprend pas la communication entre les hommes, ou qui ne voit pas les vérités de son temps, à l'opposé de cet homme d'affaires qui exploite l'artiste, nous devrions faire volte-face et contrôler ces hommes à nos propres fins, qui doivent être, à long terme, celles de la société humaine.

\section{En guise de présentation au festival de films de Pesaro, 1965}

Le dialogue entre les Critiques et les cinéastes aux Etats-Unis est insignifiant. Le nouveau cinéma, que l'on peut diviser en deux catégories, les films à bas budget comme The Connection [Shirley Clarke, I96I], 
Guns of the Trees [Jonas Mekas, I962], Hallelujah the Hills [Adolfas Mekas, I963], et le cinéma d'avant-garde, n'a reçu aucun soutien des critiques; ceux-ci ont manifesté une opposition à son égard typique d'un état d'esprit conservateur vis-à-vis de toute nouveauté.

Les longs métrages indépendants à petit budget ont obtenu un succès considérable à l'étranger. Ceci ne résout pas pour autant la question de la survie économique de leurs réalisateurs respectifs, étant donné que le plus large public qu'un film peut rencontrer se trouve dans son pays d'origine.

Les critiques new-yorkais se sont révélés constituer un obstacle plutôt qu'un appui, à cause de leur manque de sympathie envers les idées et les visées de ces cinéastes. Le problème est qu’il y a peu de spécialistes du cinéma sérieux parmi les critiques, en particulier dans la presse quotidienne. Les magazines et les revues se sont améliorés, mais ont moins d'influence sur les spectateurs. Ces critiques se préoccupent plus de l'approbation de leurs opinions par les lecteurs qu'à proposer une analyse approfondie d'un film. Leurs rubriques se réduisent justement à de simples rubriques, multipliant les potins, les anecdotes amusantes et attrayantes sur les réalisateurs et les stars. Seule leur importe leur popularité auprès des lecteurs; ils se conforment à la mentalité du grand public. Certains d'entre eux ont même avoué qu'ils doivent se montrer prudents à l'égard de films que leur lectorat n'apprécierait assurément pas - ils doivent ainsi faire attention à ne pas les recommander trop vivement pour éviter l'irritation de leurs lecteurs. Ils essaient d'être plein d'esprit et captivants plutôt qu'analytiques. Ce qui aggrave les choses, un critique en particulier détient un pouvoir presque absolu; son opinion a plus de poids que celles de tous les autres réunis en vue du succès ou de l'échec d'un soi-disant film «d'art» ${ }^{13}$.

On trouve une illustration des difficultés des cinéastes indépendants et du manque de soutien de la part des critiques dans une interview conduite par Jonas Mekas ${ }^{14}$ :

«Le film de Benjamin Manaster et Philip Kaufman Goldstein [EtatsUnis, 1964] est programmé au Fifth Avenue Cinema ${ }^{15}$. J'ai tenu la conversation suivante avec Benjamin Manaster:

Q. Combien a coûté Goldstein?

R. Moins de 50000 dollars.

Q. Est-ce que vous allez récupérer l’argent investi?
13 Rogosin fait ici probablement allusion à Bosley Crowther, critique de cinéma au New York Times depuis 1940, considéré comme très influent. Crowther avait publié en 1957 une critique virulente d'On the Bowery, qui, d'après lui, ne faisait qu'exposer de «bonnes photographies d'alcooliques et de clochards, scrutés et écoutés ad nauseam » et dont la narration n'était pas crédible («Documentary at $55^{\text {th }}$ Street Playhouse Offers Sordid Lecture on Temperance», The New York Times, 19 mars 1957, p. 44 [ma trad.]). Dans une lettre adressée à Arthur Mayer peu de temps après, Frances Flaherty explique qu'elle a reçu un appel de Rogosin, "très perturbé par la critique négative du film de Bosley Crowther, pensant qu'elle détournait les gens des projections au $55^{\text {th }}$ Street Playhouse», le cinéma dans lequel le film était alors programmé (Brattleboro [Vermont], 2 avril 1957, ALR [ma trad.]).

14 Rogosin cite ci-après la rubrique de Jonas Mekas dans I'hebdomadaire newyorkais The Village Voice ("Movie Journal», 20 mai 1965, p. 19). La retranscription est fidèle - excepté une erreur orthographique (voir note 17), mais les deux premières questions de l'entretien concernant la coréalisation du film en question ont été coupées.

15 Notons que ce film, produit et tourné à Chicago, est monté par Adolfas Mekas, le frère de Jonas, dont le film Hallelujah the Hills, en particulier son comique absurde et la construction syncopée de son récit, a été une inspiration pour Goldstein. Ben Carruthers, acteur dans Shadows de John Cassavetes (deux versions, 1958 et 1959) et Guns of the Trees de Jonas Mekas (1962), tient le rôle d'un beatnik voleur. Le film a été sélectionné à La Semaine de la Critique au Festival de Cannes, en 1964, et a partagé le Prix de la nouvelle critique avec Prima della rivoluzione de Bernardo Bertolucci (It., 1964). 
16 L'une des protagonistes apprend qu'elle est enceinte au début du film; deux médecins excentriques l'avortent au milieu du film. La promotion de Goldstein a notamment été construite autour de cette scène, comme en atteste le texte d'une publicité dans le New York Times: «Dans aucun autre film vous ne trouverez une scène aussi brute et dingue que cette scène qui brise un tabou dans Goldstein» (14 mai 1965, p. 42 [ma trad.]).

17 Son nom, correctement orthographié par Mekas, est mal retranscrit ici : il s'agit de Judith Crist. Elle est alors critique cinématographique au New York Herald Tribune.

18 Dans sa chronique de la semaine précédente, Mekas s'en prenait aux «vieux» critiques, parmi lesquels Crist et Crowther, suite à leurs recensions négatives de Goldstein et d'Across the River (Stefan Sharff, E.-U., 1965), deux films à petits budgets réalisés par de jeunes cinéastes: «Soit vous êtes un Sidney Lumet, ou alors ne faites pas de films - voilà le conseil du New York Times aux jeunes réalisateurs. [...C]es critique sont des vieux grincheux, et personne n'aime les vieux grincheux qui détestent tout ce qui n'est pas de leur âge. Néanmoins, comme l'a dit K., nous les enterrerons." ("Movie Journal», The Village Voice, 13 mai 1965, p. 19 [ma trad.]).

19 Critique cinématographique au New York Post.

20 Manaster fait référence ici à la critique d'Andrew Sarris, qui écrit en effet: « J'avais envie d'aimer Goldstein, mais le film dans son ensemble m'a laissé froid.» ("Films», The Village Voice, 6 mai 1965, p. 22 [ma trad.]).

21 L'écrivain Nelson Algren, de Chicago, joue son propre rôle dans une scène du film. Lorsque I'un des personnages lui rend visite dans son appartement, Algren lui ra-

R. Si c'est le cas, ce sera grâce à la scène d'avortement ${ }^{16}$. Les gens n'arrêtent pas d'appeler le cinéma, ils veulent savoir quand cette scène intervient, et ils viennent juste pour la voir - parce que les critiques l'ont détestée.

Q. Qu'en est-il des critiques?

R. L'un des aspects les plus déprimants des critiques de cinéma newyorkais est que si peu d'entre eux semblent saisir la structure d'ensemble du film. Le film suit un ordre primitif et une structure par épisodes qui le distingue des films conventionnels. Cet ordre est déterminé par le contenu du film, par ses images, sa musique, et ses personnages - par ce qu'ils disent et font. Il y a tellement de façons différentes à travers lesquelles ceci est montré au public qu'il est difficile de comprendre pourquoi la plupart des critiques ne l'ont pas du tout remarqué. Deux critiques, Judith Christ [sic $\left.{ }^{17}\right]$...

Q. Ne mentionne pas ce nom! $!^{18}$

R. ... Judith Christ [id.] et Archer Winsten ${ }^{19}$ ont émis beaucoup de commentaires intéressants et favorables sans pour autant adhérer au film dans son intégralité. A cet égard, j’apprécie leur intention d'analyser le film du mieux qu'ils le peuvent, malgré leurs propres réserves personnelles. Certains critiques ont été refroidis par la scène. D’autres ont semblé ne pas aimer le simple fait que nous ayons réalisé ce film. La recension dans le [Village] Voice était légitime, hormis le fait que le critique dit qu'il aurait voulu aimer le film, mais qu'il n'a simplement pas réussi ${ }^{20}$

Q. Ceci m'arrive très souvent aussi.

R. Le critique du Voice n'a pas vu le film, ou pas plus de cinq minutes. A la lecture des critiques de cinéma, j’ai l'impression générale que les critiques ont arrêté d'aller au cinéma il y a quinze ans. Goldstein est un film qui montre plus qu'il ne raconte; c'est un changement dans le cinéma que le public semble accepter plus aisément que les critiques. Ceux-ci ont aimé les scènes bavardes, comme celle avec Nelson Algren ${ }^{21}$ : pour nos critiques cinématographiques, un mot vaut plus que mille images.

Q. Quelle est d'après toi la fonction du critique de cinéma?

R. Regarder le film et tenter d'apprécier ce qui se déroule à l'écran de l'intérieur, dans les limites de ses capacités.

Q. Il me semble que c'est ce qu'ils font. Le seul problème est que l'«intériorité» à laquelle ils ont accès est ridiculement petite.

conte une histoire, durant environ cinq minutes, manifestant la mauvaise conscience du protagoniste. Visuellement, la scène est construite sur une alternance de plans rap- prochés sur Algren et son interlocuteur, et d'autres sur la bibliothèque de l'écrivain, et les bibelots et images qui la décorent. 
R. Sur ce point, on ne peut pas faire grand-chose.

Q. A Sparte, ils fracassaient la tête des bébés sur la pierre s'ils pensaient qu'ils allaient rester ridiculement petits. Bref, nos critiques de film sont tous devenus adultes maintenant, et nous ne sommes pas à Sparte. Quels films aimes-tu, toi?

R. Buñuel, Antonioni, Hallelujah the Hills, Truffaut, Godard, Fellini, Varda, et tous les autres bons réalisateurs.

Q. J'espère que tu n'es pas trop déçu du fait que ton film n’a pas été bien compris par certaines personnes.

R. Goldstein est un film qui peut être vu plus d'une fois, et il suscitera d'autres réactions lors de séances ultérieures. Je ne crois pas qu'un film qui a nécessité du temps et de la réflexion lors de sa conception doive être compris en tout point en une seule séance.»

La plupart des films indépendants sont réalisés à New York et la production a atteint un pic ces cinq dernières années. Une brève esquisse de l'histoire de ce mouvement illustrera les principaux problèmes auxquels ces personnes font face.

Il y a environ cinq ans, en i960, Jonas Mekas et moi-même avons réuni un groupe de réalisateurs afin de former une organisation dont le but était de faciliter et de réduire les coûts inhérents aux tâches insurmontables de la distribution, de la promotion, etc., de films ${ }^{22}$. Cette organisation a échoué car les membres individuels étaient trop concernés par leur désir d'obtenir des financements, et n’avaient pas pour but d'élaborer un plan commun pour distribuer ces films. Les cinéastes rencontrent trop de problèmes individuels, de tempérament et de nature artistique, pour travailler ensemble au sein d'un groupe. A mon avis, l'enjeu serait aujourd'hui de trouver un homme d'affaires assez imaginatif pour diriger une telle organisation. Les producteurs et les distributeurs entrent inévitablement en conflit par rapport à leurs propres buts et énergie, et ne parviennent pas à consacrer assez de temps à de telles organisations collectives.

Peu de temps après avoir fondé notre organisation, j’avais la conviction qu'elle n'atteindrait pas ses buts. Ma prédiction s'est révélée exacte, mais Jonas Mekas a continué tout seul à mettre sur pieds de nouvelles méthodes très précieuses pour montrer et distribuer ces nouveaux films. Il a fondé la [Filmmakers'] Cinematheque qui programme des films d'avant-garde. Ils ont déménagé d'un cinéma à un autre, errant dans New York, mais ils ont formé un public et réussi à obtenir des revenus
22 Rogosin fait ici référence à la fondation du New American Cinema Group. Notons que d'après l'introduction à la «première déclaration » du groupe publiée dans Film Culture, c'est à I'invitation de Lewis Allen et Jonas Mekas que la réunion a eu lieu ("The First Statement of the New American Cinema Group», n²2-23, été 1961, p. 130). 
23 Il s'agit de la Filmmakers' Cooperative, qui est officiellement créée en janvier 1962 (et qui est toujours active aujourd'hui).

24 II s'agit d'une rue dans le quartier de Soho, à Londres, qui hébergeait alors le siège de compagnies de production et de distribution cinématographiques.

25 En l'occurrence, la compagnie de distribution Films de France.

26 Au cinéma La Pagode, géré alors par Yvonne Decaris. indispensables pour leurs cinéastes. En outre, ils ont mis en place une coopérative qui s'occupe de la distribution $\mathrm{i} 6 \mathrm{~mm}$ de ces films ${ }^{23}$. Leur succès a été extraordinaire et a ouvert la voie à de nouvelles méthodes révolutionnaires à la fois par rapport aux techniques de réalisation et aux moyens d'exploitation et de distribution. Ces films ont été réalisés avec des sommes d'argent incroyablement réduites - aussi peu que $200 \mathrm{dol}$ lars pour des courts métrages, et 6000 dollars pour un long métrage. Avec ces coûts, nous approchons d'un idéal où le cinéaste, libéré des inhibitions liées à l'argent, pourra exprimer sa vision absolue et purement personnelle.

A mon avis, ce phénomène est tout à fait unique et révolutionnaire; il n'existe pour le moment qu'à New York. L'émergence d'une telle organisation en d'autres lieux serait bien entendu bienvenue, puisqu'un mouvement international de cette nature peut renforcer les efforts de chaque groupe individuel. Plus on développera ces activités, plus il sera facile pour les cinéastes de travailler ainsi. Une nouvelle plateforme est nécessaire et l'a été depuis de nombreuses années pour ces films - et ceci ne peut se faire qu'à un niveau international, le revenu d'un seul pays n'étant pas assez important.

Ma propre expérience avec la distribution pour Come Back, Africa et On the Bowery m'a enseigné qu'il est tout à fait irréaliste pour un réalisateur indépendant de distribuer ses propres films pays par pays. Les coûts de distribution pour un seul film sont trop importants par rapport au revenu qui peut en être tiré, et seuls des films avec un énorme potentiel économique sont recherchés par les grandes sociétés de distribution mondiale.

Après avoir terminé On the Bowery, j’ai amené le film en Angleterre et ai passé deux mois à Wardour Street ${ }^{24}$ à essayer de le vendre à un distributeur. Ce fut une tentative tout à fait décourageante. J'ai rencontré presque tous les distributeurs et ai finalement vendu le film pour une somme d'argent réduite. Je suis ensuite parti pour Paris et ai recommencé la même procédure, avec moins d'enthousiasme. Tandis que j'étais à Paris, j'ai reçu un mot des distributeurs londoniens qui annonçait qu'ils avaient changé d'avis. Le film a finalement été pris en charge par un distributeur encore plus modeste ${ }^{25}$ et a été présenté à Londres le printemps suivant. Quatre ans plus tard, il est sorti à Paris ${ }^{26}$. Maintenant, dix ans plus tard, j’ai conclu des marchés dans une grande partie de 
l'Europe et de l'Amérique du Nord. A partir de cette expérience, j’ai compris qu'il existait assurément des ressources financières assez importantes à travers le monde pour couvrir les coûts de production de films à petit budget. On ne peut toutefois obtenir ces ressources qu'à travers une organisation internationale, le producteur indépendant n'ayant pas à couvrir tous les frais lui-même.

Le nOUVEaU Groupe À New York a de nombreux objectifs. L'un d'entre eux est de développer pour ces films un circuit de distribution dans les universités, et d'obtenir plus de lieux de projection dans les grandes villes des Etats-Unis. Je me suis moi-même rendu compte à quel point le public potentiel était considérable quand mon film On the Bowery a été projeté à l'Université du Witwatersrand de Johannesburg ${ }^{27}$. Nous avons eu un public de plusieurs milliers de spectateurs sur trois soirées. J'ai ensuite reconduit une opération similaire dans trois universités américaines. Chacune des trois séances a été un grand succès.

Je suis absolument convaincu qu'il existe un marché important pour le nouveau cinéma mais qu’il doit être organisé internationalement. Au rythme où cela se faisait en i960, j’avais prédit que cela prendrait au moins vingt ans; mais avec le succès du nouveau cinéma à New York, malgré les censeurs, l'hostilité des critiques, l'apathie du public et la peur des distributeurs, il me semble qu'en cinq ans une telle organisation internationale pourrait fonctionner. Je suis convaincu que si un homme croyait en son rôle et voyageait à travers le monde avec une valise pleine de ces films, il pourrait créer une révolution dans le cinéma dans une période de cinq ans ${ }^{28}$. Non seulement au niveau de la distribution et l'exploitation, mais aussi sur le plan de la réalisation de ces films; une fois le marché créé, la production croîtra énormément.
La projection a lieu en juillet 1957.

28 Notons que c'est à peu près littéralement ce qui a été organisé par le New American Cinema Group peu auparavant: durant plusieurs mois en 1964, le jeune critique et théoricien P. Adams Sitney a présenté un programme de films associés au «New American Cinema» dans différentes villes européennes. 